\title{
The efficiency of Interatomic Coulombic Decay in Ne clusters
}

\author{
S. Barth, S. Marburger, O. Kugeler ${ }^{1}$, V. Ulrich, S. Joshi, A. M. Bradshaw, U. Hergenhahn* \\ Max-Planck-Institut für Plasmaphysik, EURATOM Association, Boltzmannstr. 2, 85748 Garching, Germany
}

\begin{abstract}
In this article, we demonstrate that Interatomic Coulombic Decay (ICD) is the dominant relaxation channel of Ne $2 s$ inner valence vacancies in free Ne clusters, with an efficiency close to $100 \%$. ICD designates a novel autoionization process of a vacancy in a weakly bonded atomic or molecular cluster. Its main characteristic is the release of an electron from a site different than the original vacancy, which is mediated by ultrafast energy transfer. Results are shown for cluster sizes between approx. 50-600 atoms. A trend towards apparently increased efficiency for larger clusters may result from inelastic scattering processes inside the cluster.
\end{abstract}

Key words: Cluster, Neon, ICD, Radiationless Decay, Interatomic Coulombic Decay

PACS: 36.40.Mr, 82.33.Fg, 34.30.+h, 33.80.Eh

\section{Introduction}

All electronically excited states decay. Whether this occurs by radiation, rearrangement of the electronic structure by emission of another electron, or a process involving the nuclear dynamics depends on the nature of the excitation, the energy in the excited state and the available final states, symmetry selection rules and a lot of other factors. For light elements excited by core level ionization, the usual relaxation pathway is Auger decay, by which the system transits from a one-hole to a local two-valence-hole state. It has been realized by L. S. Cederbaum and his coworkers that a more interesting situation occurs for light elements with a vacancy in an inner valence shell, such as the $2 s$ related orbitals in compounds of the elements Carbon to Neon. Here, the location of the double ionization potential (DIP) in the isolated atom or molecule is usually such that radiationless decay is not permitted. The presence of environmental atoms or molecules may alter this situation: Then, numerous two-hole states are conceivable, in which the two vacancies are distributed over different atoms or molecules. For inner valence vacancies, the small lowering in Coulomb repulsion due to the larger distance between the two positive holes is often enough to render autoion-

\footnotetext{
* Mailing Address: c/o BESSY mbH, Albert-Einstein-Str. 15, 12489 Berlin, Germany

Email address: uwe.hergenhahn@ipp.mpg.de (U. Hergenhahn).

1 Present Address: BESSY mbH, Albert-Einstein-Str. 15, 12489

Berlin, Germany
}

ization energetically possible [1]. This was not sufficient to conclude however, that autoionization into these states is also a viable decay route as far as the transition probability is concerned. Here again, by newly developed theoretical methods, the Cederbaum group was able to predict that the new process will have a large decay rate, and, under realistic conditions, may proceed on a femtosecond time scale $[2,3]$. The direct autoionization of an inner valence hole into a delocalized two-hole state in an extended system is now commonly termed Interatomic/Intermolecular Coulombic Decay (ICD). It is predicted to be universally present in weakly bonded systems, such as van-der-Waals or hydrogen bonded clusters, after inner valence ionization [1,3-6]. Moreover, states that may decay by ICD can form after normal (local) Auger decay of an inner shell hole [7].

The experimental investigation of ICD proved difficult in the beginning, since the electrons produced by the decay are of low kinetic energy (typically below $10 \mathrm{eV}$ ), and are therefore easily masked by electrons produced in inelastic scattering processes inside the ionized system or on apparatus parts. Nevertheless, the facts that the kinetic energy in ICD is independent of the primary ionization energy, and that ICD cannot occur below the threshold for inner valence ionization, give a handle on its isolation. Thus, some of the authors showed the existence of ICD for the Ne $2 s^{-1}$ state created by photoionization of a beam of pure Ne clusters with synchrotron radiation in 2003 [8]. One year later, a compelling investigation of ICD in the Ne dimer, based on momentum resolved coincident detection of the electron and both final state ions appeared [9]. At the same time, a 
determination of the lifetime of $\mathrm{Ne} 2 s^{-1}$ levels showed the fs time scale of ICD [10]. Another new route for investigation of these autoionization phenomena opened by the discovery of 'resonant ICD', which is the autoionization of an inner-valence excited configuration into a delocalized twohole state, in analogy to resonant Auger decay [11]. Most recent experimental works include a demonstration of ICD into mixed final states $\left(\mathrm{Ne}^{+} \mathrm{Ar}^{+}\right)$in a NeAr heterocluster [12] and the observation of ICD from doubly to triply charged clusters after conventional (local) Auger decay of Ar dimers [13].

A quantitative demonstration of the fact that ICD is the exclusive decay process for inner valence ionized levels in clusters is still missing, though. In the study reported here, by a careful analysis of electron spectra from Ne clusters with a range of sizes, we are able to demonstrate the 1:1 correspondence between the intensity of the $2 s$ photoionization, giving rise to the initial state of ICD, and the decay intensity via an ICD electron into a state with two Ne $2 p^{-1}$ vacancies.

\section{Experimental Details}

The experiments have been carried out at the undulator beamline U125/2-SGM of the synchrotron radiation source BESSY (Berlin, Germany). Descriptions of our vacuum chamber [14] and our apparatus for cluster production and electron detection [15] have been published, and will be repeated here only briefly. Some details important for the quantitative analysis of the spectra will be delineated.

A continuous beam of free clusters was produced in a supersonic expansion of Ne gas through a liquid-helium cooled conical nozzle (smallest diameter: $100 \mu \mathrm{m}$, half opening angle: $15^{\circ}$, cone length: $275 \mu \mathrm{m}$, half feeding taper angle: $45^{\circ}$ ) made from oxygen free copper. Our vacuum setup consists of an expansion chamber $\left(\mathrm{p} \approx 10^{-4}\right.$ mbar $)$ extending into the main vacuum chamber $\left(\mathrm{p} \approx 10^{-6} \mathrm{mbar}\right)$. The two volumes are separated from each other by a conical skimmer. The pressure in the expansion chamber was maintained by two turbomolecular pumps, each with a capacity of $240 \mathrm{l} / \mathrm{s}$, while in the main chamber one turbomolecular pump with a capacity of $1000 \mathrm{l} / \mathrm{s}$ evacuated the system. The spectrometer was additionally pumped at a capacity of $170 \mathrm{l} / \mathrm{s}$.

Electrons were detected with a hemispherical spectrometer (Scienta ES-200, Gammadata) mounted perpendicularly to the light propagation axis (in the dipole plane) and at an angle of $54.7^{\circ}$ (magic angle) to the electric-field vector of the synchrotron radiation.

This analyser is equipped with a multiplex electron detector consisting of a pair of microchannel plates (MCP) read out via a fluorescent screen and a charged-couple device (CCD) camera. The positions of illuminated CCD pixels are read into a computer and the spectrum vs. energy is formed by a suitable software. This approach has two known problems: 1 . Since typically more than one pixel is illuminated by a single electron detected in the analyser,
Table 1

Expansion parameters and cluster size.

\begin{tabular}{lll}
$p$ (mbar) & $T(\mathrm{~K})$ & $\langle N\rangle$ (atoms) \\
\hline 510 & 55.3 & 47.3 \\
490 & 51.1 & 65.8 \\
464 & 46.4 & 97.2 \\
431 & 41.3 & 152.9 \\
419 & 39.4 & 184.3 \\
412 & 38.2 & 209.2 \\
701 & 40.8 & 512.0
\end{tabular}

the spectrum does not conform to Poissonian statistics. 2. For some comparable instruments, the response of the fluorescence screen/camera combination was found to be nonlinear at moderate to high intensities [16]. We have corrected for both of these effects: Poissonian statistics were restored by multiplying the spectra with a constant factor (0.1) determined from the fluctuations in sections of spectra with constant count rate vs. energy. The analyser nonlinearity was corrected from calibration measurements at different synchrotron radiation intensity, but turned out to be a small correction at count rates encountered here.

The photon energy was corrected from published values for the binding energy of the $\mathrm{Xe} 4 d$ photoline and kinetic energy of one of the $\mathrm{N}_{4,5} \mathrm{O}_{2,3} \mathrm{O}_{2,3}$ Auger lines [17]. Additionally, literature values for the binding energy of some Ne $2 p^{4} \mathrm{n} l$ correlation satellites [18] were used to correct the kinetic-energy scale of the detector.

The kinetic-energy dependent transmission function of the analyser was checked by comparing the signal intensities of some Xe NOO Auger lines to the Xe $4 d$ photolines at a number of different photon energies and a correction was applied to the spectra. Measurements reported here were done with a pass energy of $5 \mathrm{eV}$ in order to have a (relatively) good transmission for low kinetic energy electrons. The transmission then drops strongly for electrons with kinetic energies below $1 \mathrm{eV}$, but at a kinetic energy of about $0.6 \mathrm{eV}$ the signal can still be analysed. The overall apparatus energy resolution of about $80 \mathrm{meV}$ was mainly determined by the analyser settings (slit width: $2.5 \mathrm{~mm}$, curved).

The drop in ring current during the acquisition time of one spectrum was about $2.2 \%$. Its correction was applied to the final result (Fig. 2).

A range of cluster sizes is produced in a supersonic expansion source. Due to the weak bonding of noble gas clusters, the correct determination of the mean cluster size $\langle N\rangle$ in dependence of the source parameters is still a topic of debate. So far, results obtained with different techniques have not entirely converged even for Ar clusters, which is the case studied best (see e.g. [19]). In this work, we will give size estimates based on the scaling law proposed by Hagena [20]:

$\langle N\rangle=33\left(\frac{\Gamma^{*}}{1000}\right)^{2.35}$, 


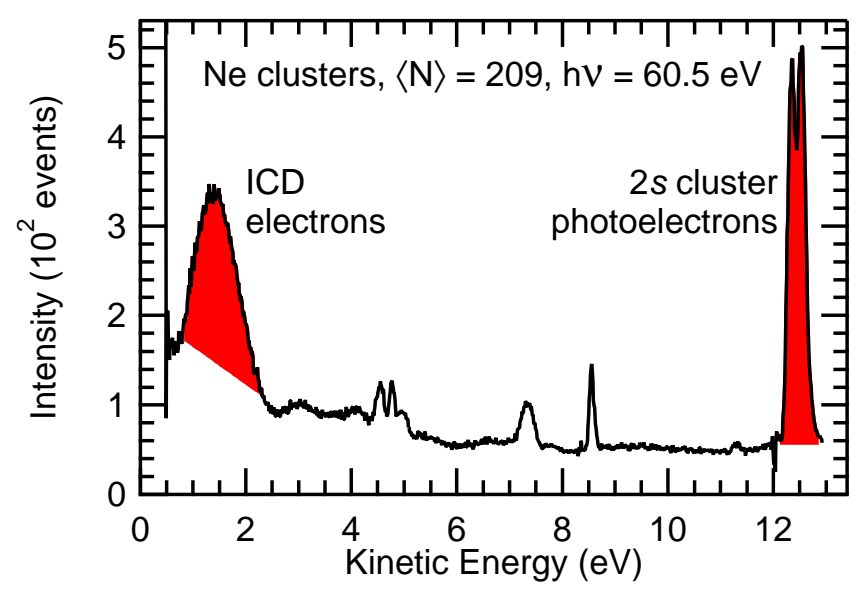

Fig. 1. (Color online) Electron intensity vs. kinetic energy of the cluster contribution. A scaled and energetically shifted monomer spectrum was subtracted from the original spectrum. The ICD and the $\mathrm{Ne} 2 s$ cluster photoline signals are represented by the shaded areas.

with the scaling parameter $\Gamma^{*}$, which reads in the form adapted by Karnbach et al. [21]:

$\Gamma^{*}=\frac{p d^{q}}{T^{5 / 2-q / 4}} k$.

Here, $p$ is the stagnation pressure, $T$ the temperature and $d$ the diameter of a flat, circular nozzle. For a conical nozzle, the 'equivalent diameter' $d_{e q}$ defined by $d_{e q}:=$ $0.736 d / \tan \alpha$, with $\alpha$ the half opening angle of the cone, has to be inserted in place of $d$. $q$ is an empirical parameter, which we have set to 0.85 following earlier work [22]. The parameter $k$ contains all properties of the material and can be calculated from the sublimation enthalpy per atom at $0 \mathrm{~K}\left(\Delta h_{0}^{0}\right)$ and a characteristic interatomic distance $r_{c h}$ calculated from the density of the solid at $0 \mathrm{~K}$ [23]. When we use $p$ in mbar, $d$ in $\mu \mathrm{m}$ and $T$ in $\mathrm{K}$ in eq. (2), we get

$k=\frac{100 \cdot\left(10^{-6}\right)^{q}}{\tilde{k} r_{c h}^{q-3}\left(\Delta h_{0}^{0} / \tilde{k}\right)^{q / 4-3 / 2}}$,

with a numerical value of $k=187.23$ using ref.s [25,24], in agreement with [21] ( $\tilde{k}$ : Boltzmann constant).

Alternatively, the size of clusters can be estimated from the bulk-to-surface ratio of a suitable photoline [26]. Using the Ne $2 s$ photoelectron signal with this method leads to cluster sizes about a factor of 2 larger than the estimate from the scaling law. We note that the short, conical geometry of our nozzle does not agree with cases investigated earlier $[22,21]$ but the actual cause of the deviations currently is open. For convenience the values of the stagnation pressure $p$, nozzle temperature $T$ and $\langle N\rangle$ are given in Table 1 .

\section{Data Analysis}

In this article, we are aiming at a quantitative determination of the efficiency of ICD as a decay channel for $\mathrm{Ne} 2 s^{-1}$ vacancy states in Ne clusters. The ICD signal from larger

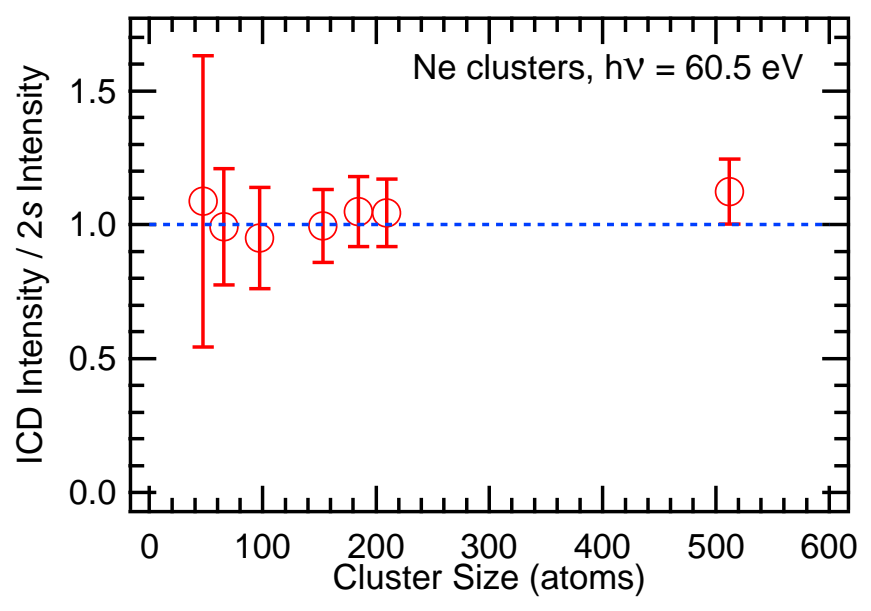

Fig. 2. (Color online) Ratio of Ne ICD intensity to Ne $2 s$ intensity as a function of mean cluster size $\langle N\rangle$. The ratio is close to unity as expected, possible reasons for the deviations for larger clusters are discussed in the text. A scale error of the vertical axis on the order of $10 \%$ is not included in the error bars (see text).

Ne clusters has been shown in earlier work [8], and is located between 0.6 and $2 \mathrm{~V}$ kinetic energy, in the form of a symmetric hump. In principle, the efficiency of ICD can be determined in a straightforward manner by comparing the intensity of this signal in an electron spectrum to the intensity of the $2 s$ photoline. However, several experimental problems make it somewhat demanding to arrive at meaningful results in practice. 1. Since the transmission function of any electron analyser varies strongly in the region of low kinetic energies, its careful determination is essential. 2. Besides ICD, in photoionization of clusters a low kinetic energy background is produced by intracluster inelastic scattering. Spurious intensity may also occur in this energy region due to apparatus effects. These factors lead to difficulties in peak-background separation for the ICD signal. 3 . The appearance thresholds of numerous correlation satellites pertaining - in an atomic language - to $2 p^{4} n l$ configurations are located at binding energies between roughly 55 and $80 \mathrm{eV}$, which is a photon energy range convenient for the desired measurements. These satellites therefore superimpose the ICD structure and have to be subtracted. A recent publication by some of the authors shows that in clusters the satellites also broaden and atomic satellite lines may split [27].

Here, we have chosen to record a series of electron spectra for a range of cluster sizes, in which stagnation pressure and nozzle temperature were varied to produce cluster sizes from nearly atomic to the maximum that was feasible at these apparatus conditions. All spectra were recorded at a photon energy of $60.5 \mathrm{eV}$.

Intensities of the ICD signal and the $2 s$ photoelectron signal were then extracted using two different methods: In the first method, areas were determined from integrating the signal between left and right border of the respective peak, while subtracting a linear background and the satellite intensities mentioned above. In the second method, a spectrum containing only monomer contributions was 
subtracted before peak area determination. This monomer spectrum was scaled to total intensity of the atomic $2 s$ component (resulting from the uncondensed part of the $\mathrm{Ne}$ beam) for each cluster size. A typical difference spectrum representing the isolated cluster signal is shown in Fig. 1. In the difference spectra, areas were then determined as explained before. Results of the two methods do not agree exactly. The difference between them is thought to give a reasonable estimate for errors in peak area determination due to e.g. ambiguities in definition of the background to the ICD peak, and is incorporated in the error bars of the results shown below (Fig. 2). Besides this contribution, error bars in Fig. 2 contain the propagation of the statistical errors. Another contribution to the experimental errors, resulting from inaccuracy of the transmission function determination, is not included in the individual error bars since it would influence all data points in the same way. This scale error can be estimated as $10 \%$, that is a measured fraction of 1.0 is expected to lie between 0.9 and 1.1 .

\section{Results and Discussion}

The intensity of the IC decay of $\mathrm{Ne} 2 s^{-1}$ states in $\mathrm{Ne}$ clusters normalized to the primary $2 s$ photoelectron signal is displayed as a function of cluster size in Fig. 2. This ratio is rather constant and close to unity for the whole range of cluster sizes investigated. For the smallest cluster sizes, the absolute intensity of the cluster signal is worse, since the beam contains mainly uncondensed atoms. Therefore, statistical error bars are larger for these cases. Ne ICD signal and Ne $2 s$ cluster photoelectron signal are of the same intensity. This leads to the clear conclusion that indeed nearly every single $2 s$ inner valence vacancy in the Ne cluster decays via ICD. ICD, as an ultrafast process, outpaces all competing decay channels, as predicted by Cederbaum and coworkers.

On further inspection, towards large cluster size there is an apparent trend of an increase in the ratio, although taking into account the error bars a constant ratio at or slightly above unity is supported by all measurements as well. Nevertheless, a slight increase might be expected from the following argumente: Both types of electrons, the slow ICD electrons as well as the faster $2 s$ photoelectrons emerge from nearly the same site of the cluster. In average they have to travel the same way through the cluster and have to pass the same number of cluster constituents on their way to the detector. Because of their different kinetic energies, however, they have different inelastic scattering cross sections. Their mean free paths differ, and are indeed shorter for faster photoelectrons [28]. This effect will lead to a decrease of the Photoelectron/ICD electron intensity ration which may well be present in our data, although we cannot prove this at a statisically significant level.

One further point deserves to be mentioned: While the theoretical and experimental energy spectrum of ICD in the Ne dimer has non-vanishing intensity even at kinetic energies infinitely close to zero eV $[9,29]$, we see a significant reduction in ICD intensity at energies below one $\mathrm{eV}$. Although the detection limit of our analyser is close to this energy, we are confident that this reduction is not solely an apparatus effect. An explanation might come from noting that in the dimer case, the lowest kinetic energies in ICD correspond to the largest kinetic energy release to the fragments in the subsequent disintegration of the cluster. These channels might be hindered in a larger cluster.

\section{Summary}

We have experimentally demonstrated that ICD (Interatomic/molecular Coulombic Decay) is the dominating decay channel for inner valence vacancies in larger Ne clusters $(\langle N\rangle=50-600$ atoms $)$. This finding has far-reaching consequences, since the same behaviour can be expected wherever ICD is energetically possible in an extended, weakly bonded system.

\section{Acknowledgments}

Thanks are due to L. S. Cederbaum and his coworkers for numerous discussions about all aspects of the investigation of ICD. The authors would like to acknowledge the effective support by the BESSY team during data acquisition. Funding by the Deutsche Forschungsgemeinschaft is gratefully acknowledged. This work was also supported by the Fonds der Chemischen Industrie.

\section{References}

[1] L.S. Cederbaum, J. Zobeley, F. Tarantelli, Phys. Rev. Lett. 79 (1997) 4778.

[2] R. Santra, J. Zobeley, L.S. Cederbaum, Phys. Rev. B 64 (2001) 245104.

[3] R. Santra, L.S. Cederbaum, Phys. Rep. 368 (2002) 1.

[4] J. Zobeley, L.S. Cederbaum, F. Tarantelli, J. Chem. Phys. 108 (1998) 9737; J. Phys. Chem. A 103 (1999) 11145.

[5] J. Zobeley, R. Santra, L.S. Cederbaum, J. Chem. Phys. 115 (2001) 5076.

[6] I.B. Müller, L.S. Cederbaum, J. Chem. Phys. 122 (2005) 094305; J. Phys. Chem. A 108 (2004) 5831.

[7] R. Santra, L.S. Cederbaum, Phys. Rev. Lett. 90 (2003) 153401.

[8] S. Marburger, O. Kugeler, U. Hergenhahn, T. Möller, Phys. Rev. Lett. 90 (2003) 203401.

[9] T. Jahnke, A. Czasch, M.S. Schöfler, S. Schssler, A. Knapp, M. Käsz, J. Titze, C. Wimmer, K. Kreidi, R.E. Grisenti, A. Staudte, O. Jagutzki, U. Hergenhahn, H. Schmidt-Böcking, R. Dörner, Phys. Rev. Lett. 93 (2004) 163401.

[10] G. Öhrwall, M. Tchaplyguine, M. Lundwall, R. Feifel, H. Bergersen, T. Rander, A. Lindblad, J. Schulz, S. Peredkov, S. Barth, S. Marburger, U. Hergenhahn, S. Svensson, O. Björneholm, Phys. Rev. Lett. 93 (2004) 173401.

[11] S. Barth, S. Joshi, S. Marburger, V. Ulrich, A. Lindblad, G. Öhrwall, O. Björneholm, U. Hergenhahn, J. Chem. Phys. 122 (2005) 241102. 
[12] S. Barth, S.P. Marburger, S. Joshi, V. Ulrich, U. Hergenhahn, Phys. Chem. Chem. Phys. 8 (2006) 3218.

[13] Y. Morishita, X.-J. Liu, N. Saito, T. Lischke, M. Kato, G. Prümper, M. Oura, H. Yamaoka, Y. Tamenori, I.H. Suzuki, K. Ueda, Phys. Rev. Lett. 96 (2006) 243402.

[14] F. Pink, G. Schmidt, Appl. Phys. A 78, (2004) 667.

[15] S.P. Marburger, O. Kugeler, U. Hergenhahn, in: T. Warwick, J. Arthur, H.A. Padmore and J. Stöhr (Ed.s) Synchrotron Radiation Instrumentation: Eighth International Conference, American Institute of Physics, 2003, p. 1114.

[16] N. Manella, S. Marchesini, A.W. Kay, A. Nambu, T. Gresch, S.-H. Yang, B.S. Mun, J.M. Bussat, A. Rosenhahn, C.S. Fadley, J. Electron Spectrosc. Relat. Phenom. 141 (2004) 45.

[17] T.X. Carroll, J.D. Bozek, E. Kukk, V. Myrseth, L.J. Sæthre, T.D. Thomas, K. Wiesner, J. Electron Spectrosc. Relat. Phenom. 125 (2002) 127.

[18] A. Kikas, S.J. Osborne, A. Ausmees, S. Svensson, O.-P. Sairanen, S. Aksela, J. Electron Spectrosc. Relat. Phenom. 77 (1996) 241.

[19] U. Buck, R. Krohne, J. Chem. Phys. 105 (1996) 5408.

[20] O.F. Hagena, Rev. Sci. Instrum. 63 (1992) 2374.

[21] R. Karnbach, M. Joppien, J. Stapelfeldt, J. Wörmer, T. Möller, Rev. Sci. Instrum. 64 (1993) 2838.

[22] O.F. Hagena, Surf. Sci. 106 (1981) 101

[23] O.F. Hagena, Z. Phys. D 4 (1987) 291.

[24] G.T. McConville, J. Chem. Phys. 60 (1974) 4093.

[25] D.N. Batchelder, D.L. Losee, R.O. Simmons, Phys. Rev. 162 (1967) 767.

[26] M. Tchaplyguine, R.R. Marinho, M. Gisselbrecht, J. Schulz, N. Mårtensson, S.L. Sorensen, A. Naves de Brito, R. Feifel, G. Öhrwall, M. Lundwall, S. Svensson, O. Björneholm, J. Chem. Phys. 120 (2004) 345.

[27] S. Joshi, S. Barth, S. Marburger, V. Ulrich, U. Hergenhahn, Phys. Rev. B 73(2006) 235404.

[28] N. Schwentner, Phys. Rev. B 14 (1976) 5490.

[29] S. Scheit, V. Averbukh, H.-D. Meyer, N. Moiseyev, R. Santra, T. Sommerfeld, J. Zobeley, L.S. Cederbaum, J. Chem. Phys. 121 (2004) 8393. 